\title{
Altered corticospinal microstructure and motor cortex excitability in gliomas: an advanced tractography and transcranial magnetic stimulation study
}

\author{
*Ayesha Sunil Mirchandani, MBBS, MSc, ${ }^{1}$ Ahmad Beyh, MSc,, José Pedro Lavrador, MD, ${ }^{3}$ \\ Henrietta Howells, PhD, ${ }^{2}$ Flavio Dell'Acqua, PhD, ${ }^{2}$ and Francesco Vergani, PhD, FRCS ${ }^{3}$
}

${ }^{1}$ Neuroimaging Department, King's College London; ${ }^{2}$ NatBrainLab, Department of Forensics and Neurodevelopmental Sciences, King's College London; and 'Neurosurgical Department, King's College London Hospital, London, United Kingdom

\begin{abstract}
OBJECTIVE This prospective case-control study was conducted to examine whether spherical deconvolution (SD) can unveil microstructural abnormalities in the corticospinal tract (CST) caused by IDH-mutant gliomas. To determine the significance of abnormal microstructure, the authors investigated the correlation between diffusion parameters and neurophysiological data collected with navigated transcranial magnetic stimulation (nTMS).

METHODS Twenty participants (10 patients and 10 healthy controls) were recruited. Diffusion-weighted images were acquired on a 3-T MRI scanner using a cardiac-gated single-shot spin echo echo-planar imaging multiband sequence (TE $80 \mathrm{msec}$, TR $4000 \mathrm{msec}$ ) along 90 diffusion directions with a b-value of $2500 \mathrm{sec} / \mathrm{mm}^{2}$ (FOV $256 \times 256 \mathrm{~mm}$ ). Diffusion tensor imaging tractography and SD tractography were performed with deterministic tracking. The anterior portion of the ipsilateral superior peduncle and the precentral gyrus were used as regions of interest to delineate the CST. Diffusion indices were extracted and analyzed for significant differences between hemispheres in patients and between patient and control groups. A navigated brain stimulation system was used to deliver TMS pulses at hotspots at which motor evoked potentials (MEPs) for the abductor pollicis brevis, first digital interosseous, and abductor digiti minimi muscles are best elicited in patients and healthy controls. Functional measurements such as resting motor threshold (rMT), amplitude of MEPs, and latency of MEPs were noted. Significant differences between hemispheres in patients and between patients and controls were statistically analyzed. The Spearman rank correlation was used to investigate correlations between diffusion indices and functional measurements.
\end{abstract}

RESULTS The hindrance modulated orientational anisotropy (HMOA), measured with SD tractography, is lower in the hemisphere ipsilateral to glioma $(p=0.028)$. The $\mathrm{rMT}$ in the hemisphere ipsilateral to a glioma is significantly greater than that in the contralateral hemisphere $(p=0.038)$. All measurements contralateral to the glioma, except for the mean amplitude of MEPs $(p=0.001)$, are similar to those of healthy controls. Mean diffusivity and axial diffusivity from SD tractography are positively correlated with rMT in the hemisphere ipsilateral to glioma ( $p=0.02$ and 0.006 , respectively). The interhemispheric difference in HMOA and rMT is correlated in glioma patients $(p=0.007)$.

CONCLUSIONS SD tractography can demonstrate microstructural abnormality within the CST of patients with IDH1mutant gliomas that correlates to the functional abnormality measured with nTMS.

https://thejns.org/doi/abs/10.3171/2020.2.JNS192994

KEYWORDS low-grade gliomas; pathological excitability; tractography; spherical deconvolution; resting motor threshold; transcranial magnetic stimulation; corticospinal tract; oncology

$\mathrm{G}$ LIOMAS in proximity to the motor cortex and corticospinal tract (CST) represent a challenge to neurosurgeons, as the risk of inducing permanent motor deficits is high. ${ }^{1,2}$ Intraoperative mapping and monitoring remain the gold standard to assess the integrity of the mo- tor system. ${ }^{3}$ However, in recent years, there has been interest in preoperatively delineating the anatomy and function of the primary motor cortex and CST.4,5 Preoperative imaging of the CST is useful for planning glioma resection. ${ }^{6}$ The relationship of tumors with the CST and motor cortex

ABBREVIATIONS $A D=$ axial diffusivity; $C S T$ = corticospinal tract; $D T I=$ diffusion tensor imaging; $F A=$ fractional anisotropy; fODF = fiber orientation distribution function; $\mathrm{HMOA}=$ hindrance modulated orientational anisotropy; MD = mean diffusivity; MEP = motor evoked potential; MRC = Medical Research Council; nTMS = navigated transcranial magnetic stimulation; $\mathrm{RD}=$ radial diffusivity; $\mathrm{rMT}$ = resting motor threshold; $\mathrm{ROI}=$ region of interest; $\mathrm{SD}=$ spherical deconvolution.

SUBMITTED November 4, 2019. ACCEPTED February 21, 2020.

INCLUDE WHEN CITING Published online May 1, 2020; DOI: 10.3171/2020.2.JNS192994.

${ }^{*}$ F.D. and F.V. contributed equally to this work. 
can impact the extent of resection, occurrence of new motor deficits, and long-term recovery after surgery. $5,7,8$

The CST can be radiologically assessed using diffusion MRI tractography. In tractography, information about the directional variance (anisotropy) of water diffusion is used to estimate mathematical reconstructions (trajectories) of white matter tracts. Diffusion imaging parameters include measures of the degree of anisotropy (fractional anisotropy [FA]), average displacement during diffusion (mean diffusivity [MD]), and average displacement in the radial and axial axes during diffusion (axial diffusivity [AD] and radial diffusivity [RD]). Traditional tractography is, however, limited by crossing fibers and the partial volume effects. ${ }^{9}$

Traditional tractography parameters have yielded conflicting results when used to assess white matter connections in glioma patients. It is unclear, for example, whether these parameters represent axonal integrity ${ }^{10}$ and there is inconsistent evidence about their variation between highgrade and low-grade gliomas. ${ }^{11,12}$ Finally, there is no consensus about the strength of their correlation to clinical motor function measures. ${ }^{13,14}$

Advanced tractography methods such as spherical deconvolution (SD) estimate multiple fiber orientations per voxel. SD uses a continuous fiber orientation distribution function (fODF) to describe the amplitude (density) and orientation of multiple white matter fibers in each voxel. This function is derived from diffusion MRI data deconvolved with a model of white matter fiber (fiber response function). One of the widely used deconvolution methods is the damped Richardson-Lucy (dRL) SD.$^{15}$ This method accurately resolves crossing fibers even in noisy data and preserves the angular resolution of main fiber orientations even in the presence of partial volume contamination..$^{15,16}$ Moreover, advanced tractography parameters, such as measures of the hindrance modulated orientational anisotropy (HMOA), are tract specific and can be used as a proxy for fiber density and individual fiber anisotropy ${ }^{16,17}$

Navigated transcranial magnetic stimulation (nTMS) has been introduced in the last decade to noninvasively map the primary motor cortex for presurgical planning in patients with brain tumors. ${ }^{5,18-20}$ By stimulating the primary motor cortex, motor evoked potentials (MEPs) are elicited in peripheral muscles, and their latency and amplitude can be estimated. nTMS is also useful in defining the resting motor threshold (rMT) of the primary motor cortex. Differences in the rMT between the pathological and healthy hemisphere have been used to assess the presence of a "pathological excitability" of the motor cortex (defined as an rMT difference of at least $10 \%$ between the healthy and pathological brain hemisphere). Patients with a pathological excitability are at higher risk of developing motor deficits after surgery. ${ }^{5}$

It is unclear whether any correlation exists between changes observed in the CST with tractography indices (including "traditional" and advanced parameters such as HMOA) and the presence of pathological excitability or altered MEP values. This is relevant, as at present we do not know whether changes seen with tractography in the CST have a neurophysiological correlate. In the current study, we assessed the microstructural quantification of the CST in healthy volunteers and in glioma patients using traditional diffusion tensor imaging (DTI) tractography and relative metrics (FA, MD, AD, RD) compared to SD tractography and tract-specific metrics (HMOA). We also correlated changes in diffusion measures with neurophysiology findings obtained with nTMS (MEP and rMT values and the presence or absence of pathological excitability of the motor cortex).

\section{Methods \\ Patient and Healthy Control Sample}

Twenty participants were recruited into this singlecenter prospective cohort study. Ten patients (4 females, 6 males; mean age 41 years, range 28-57 years) had a histologically confirmed diagnosis of infiltrative diffuse glioma involving or near the central lobule (precentral and postcentral gyri). Patients with previous surgery, multiple brain lesions, a WHO performance status of 2 or more, a histological diagnosis other than glioma, or an age below 18 or above 60 were not included. Ten healthy controls (4 females, 6 males; mean age 31 years, range $24-42$ years) were closely matched to the patients with regard to sex and age. Healthy controls were screened for neurological and psychiatric history before inclusion. All patients and healthy controls were right-handed. Recruitment and data acquisition were approved by the South Yorkshire Research Ethics Committee, and written informed consent was obtained from all participants.

\section{MRI Study Acquisition}

A 3-T MR750 MRI scanner (General Electric) was used to acquire imaging data. Diffusion data were acquired using a cardiac-gated single-shot spin echo echoplanar imaging multiband sequence and 32-channel head coil (Nova Medical) with the following parameters: 75 axial slices, TE $80 \mathrm{msec}$, TR 5 R-R interval (approximatively $4000 \mathrm{msec}$ ), acquisition matrix $128 \times 128$, and FOV $256 \times 256 \mathrm{~mm}^{2}$. Data were collected along 90 diffusion directions with a b-value of $2500 \mathrm{sec} / \mathrm{mm}^{2}$. A total of $18 \mathrm{~b}$ $=0$ diffusion weighting volumes were collected, 12 with anterior-to-posterior (AP) phase orientation and 6 with reversed posterior-to-anterior (PA) phase polarity for susceptibility correction. A multiband acceleration factor of 3 and an in-plane acceleration factor ARC (autocalibrating reconstruction for Cartesian imaging) of 2 were used. Diffusion data were de-noised ${ }^{21}$ and corrected for Gibbs ringing, ${ }^{22}$ motion, ${ }^{23}$ eddy currents, ${ }^{24}$ and susceptibility distortion. ${ }^{24}$ Outlier slices were then detected and replaced. ${ }^{24}$ Finally, intravolume slice motion was corrected for. ${ }^{25}$ Data from one patient were incomplete and therefore excluded from analysis.

\section{Tractography Reconstruction}

Neuroimaging analysis was completed by NatBrainLab members (A.S.M. and A.B.) under the supervision of the senior author (F.D.). StarTrack software was used for traditional single tensor tractography (DTI) and advanced SD tractography (www.natbrainlab.com). Wholebrain DTI tractography used deterministic tracking (Euler streamline propagation with a step size of $1 \mathrm{~mm}$ ). Included 
trajectory streamlines were no shorter than $20 \mathrm{~mm}$ and no longer than $30 \mathrm{~mm}$. Tracking was terminated if there was deviation any greater than $25^{\circ}$ or FA below 0.15 . The SD modeling was performed using the dRL algorithm (fiber response parameter ALFA $=1.5,300$ iterations, regularization $\eta=0.0015, v=16$ ). Deterministic tracking was performed with an angle threshold of $35^{\circ}$, an absolute fODF amplitude threshold of 0.002 , and a step size of 1 $\mathrm{mm}$. Seeding was set to 1 seed per fiber orientation.

The CST was dissected using regions of interest (ROIs) previously described: anterior portion of the ipsilateral superior peduncle, precentral gyrus, and CST area in the brainstem. ${ }^{26,27}$ Freehand ROIs were outlined on 2D FA maps on TrackVis to select fibers from specific anatomical regions through which the CST passes. Exclusion ROIs were created based on prior anatomical knowledge to eliminate artifactual streamlines. Trajectories were checked by the senior author (F.D.).

Measures of microstructure were averaged across all voxels through which the CST passed. For all CST trajectories (both DTI and SD), mean FA, MD, AD, and RD were extracted. In addition, for trajectories reconstructed by SD, the HMOA (absolute amplitude of each lobe in the fODF) was extracted. The maximum HMOA is averaged across all voxels through which the CST passes and used as a proxy for fiber density and FA in SD trajectories.

\section{Transcranial Magnetic Stimulation}

The eXimia navigated brain stimulation system (Nexstim) was used for preoperative motor mapping. A figure-8 coil was used to deliver a TMS pulse. The electric field was estimated based on a dynamic spherical model adjusted in real time. The lowest stimulation intensity (percentage of maximum stimulator intensity) capable of eliciting an MEP in 50\% of trials for the hand area in both brain hemispheres was used to calculate the rMT. MEPs were recorded from the abductor pollicis brevis, first digital interosseous, and abductor digiti minimi muscles. The mean amplitude and mean latency of MEPs of the tested muscles were measured. TMS mapping (not blinded) was performed by two operators (F.V. and H.H.). A pathological excitability was considered to be present when the interhemispheric rMT ratio (irMTr) was larger than $10 \%$ (irMTr $<0.90$ or $>1.10) .{ }^{12}$

\section{Tumor Resection}

Tumor removal was performed with the aim of maximal resection according to anatomical and functional boundaries. Neuronavigation (StealthStation S7, Medtronic) was employed in all cases. The compound 5-aminolevulinic acid (5-ALA) was used as an adjunct when enhancement was present on preoperative MRI. Intraoperative motor mapping with a constant current stimulator and a monopolar probe (ISIS Xpress system, Inomed $\mathrm{GmbH}$ ) was performed using train of 5 stimulations with a 0.5 -msec pulse width and interstimuli interval of $4.0 \mathrm{msec}$. Cortical mapping was performed with positive pulse form, and the subcortical mapping was performed with negative pulse form. Motor responses were recorded from the face, upper limb/ hand, and/or lower limb/foot, according to tumor location and extent of motor cortex exposure. The monitoring of the integrity of the CST was performed with continuous MEPs elicited from a 4-contact subdural strip electrode placed over the precentral gyrus (best contact of the 4). Placement of the strip was guided by the cortical mapping or by the nTMS preoperative mapping when the primary motor cortex was not exposed. The extent of resection was calculated as follows, based on the postoperative MRI study obtained within 48 hours from surgery: gross total (no residual tumor visible on postoperative MRI), subtotal (residual volume 1-10 ml), and partial (residual volume more than $10 \mathrm{ml}){ }^{28}$ The residual volume was calculated based on the residual hyperintensity visible on postoperative T2/FLAIR sequences.

\section{Statistical Analysis}

SPSS software (version 25, IBM Corp.) was used for statistical analysis. Prior to hypothesis testing, the distribution of diffusion indices and rMTs were assessed with the Shapiro-Wilk test. For all statistical tests, a significance threshold of 0.05 was used. We tested the hypothesis that microstructure and TMS indices are significantly different between cerebral hemispheres ipsilateral and contralateral to a glioma. Relative differences of indices from trajectories ipsilateral and contralateral to the tumor were calculated. The Wilcoxon signed-rank test was used to determine if the difference is significant. This hypothesis assumes that measurements contralateral to the glioma are within the healthy range. The assumption was tested using the Kolmogorov-Smirnov test. It was also used to test for asymmetry between the right and left hemispheres in the healthy data set. This test was chosen because it does not assume that the data are homoscedastic.

The hypothesis that functional (nTMS) and anatomical (tractography) measurements correlate was tested using the Spearman rank order correlation, which was used because it does not assume that the data are normally distributed.

\section{Results}

\section{Patient Results}

Histological analysis for the 10 patients was consistent with WHO grade II (1 case) and III (9 cases) gliomas; all 10 cases were $I D H I$ mutant. There were 5 astrocytomas and 5 oligodendrogliomas (1p/19q codeletion confirmed). Seventy percent of the patients had a glioma within $1 \mathrm{~mm}$ of the CST as assessed on preoperative tractography. All patients had a Medical Research Council (MRC) grade $5 / 5$ preoperatively. Thirty percent of the patients had gross-total resection, 50\% had subtotal resection, and 20\% had partial resection. One patient suffered a postoperative motor deficit (MRC grade 4/5 in the right upper limb) that did not improve by the 6-month follow-up. This was possibly the result of a postoperative brain abscess that had been surgically drained. Two patients (20\%) suffered from postoperative motor seizures. Patient characteristics are described in Table 1.

The mean intraoperative rMT for the hand area was 8.9 $\mathrm{mA}$ (range 4-14 mA). We observed a degree of correlation between intraoperative rMT and TMS rMT (with a higher 
TABLE 1. Summary of 10 glioma patient characteristics

\begin{tabular}{cc}
\hline \multicolumn{1}{c}{ Variable } & No. $(\%)$ \\
\hline Sex & \\
\hline Female & $4(40)$ \\
\hline Male & $6(60)$ \\
\hline Age in yrs & 41 \\
\hline Mean & $28-57$ \\
\hline Range & \\
\hline Diagnosis & $1(10)$ \\
\hline WHO grade II & $9(90)$ \\
\hline WHO grade III & $10(100)$ \\
\hline IDH mutation & $2(20)$ \\
\hline Complication & $1(10)$ \\
\hline Seizures & $10(100)$ \\
\hline Infection & $1(10)$ \\
\hline Preop MRC grade $5 / 5$ & $9(90)$ \\
\hline Postop MRC grade & \\
\hline 4/5 & $3(30)$ \\
\hline $5 / 5$ & $5(50)$ \\
\hline Extent of resection & $2(20)$ \\
\hline GTR & \\
\hline STR & \\
\hline Partial & \\
\hline
\end{tabular}

GTR = gross-total resection; STR = subtotal resection .

rMT on TMS generally corresponding to a higher rMT intraoperatively), although the relationship was not significant. Mean subcortical stimulation was $5.4 \mathrm{~mA}$. Fibers of the CST were identified in 7 patients using subcortical stimulation, which guided resection.

\section{Tractography Results}

Qualitative analysis of CST trajectories from DTI and SD revealed that in $60 \%$ of the cases, SD tractography reconstructs a thicker trajectory through the glioma/edematous regions. In all of the reconstructions, SD tractography reconstructed trajectories that more closely resembled the anatomical fanning shape of the CST at its cranial end (Fig. 1).

Diffusion indices ipsilateral and contralateral to a glioma are illustrated in Figs. 2 and 3. The differences in all traditional tractography measures (FA, MD, AD, and RD) between the hemisphere with a glioma and the contralateral hemisphere are not statistically significant using both SD and DTI ( $p>0.05)$.

The tract-specific index HMOA is significantly lower in the hemisphere ipsilateral to glioma (median relative difference 5.2\%, IQR $\pm 7.9 \%$; $p=0.028$; Fig. 3 ).

Finally, diffusion indices of the CST contralateral to glioma are not significantly different from those of healthy brains on DTI and SD (Table 2).

Further analysis was performed to assess if left and right asymmetry is a significant contribution to our results. Statistical analysis revealed no significant differences between left and right brain hemispheres for FA, MD, AD,

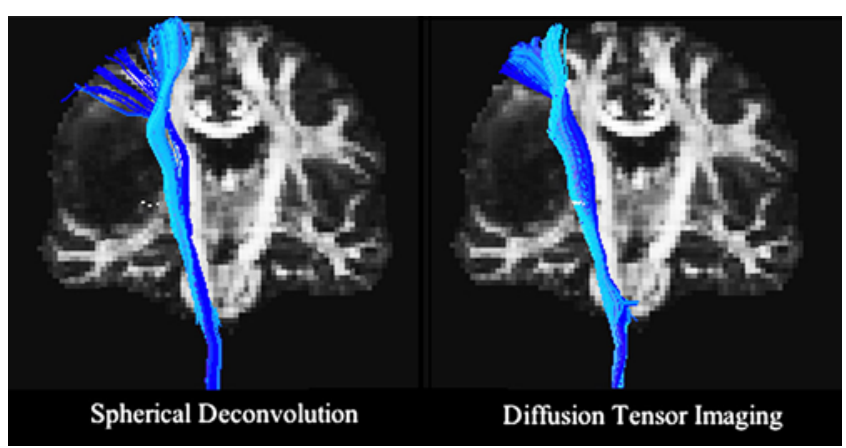

FIG. 1. Reconstructions of the CST using SD and DTI at a diffusion weighting of $b=2500 \mathrm{sec} / \mathrm{mm}^{2}$ on an FA map. Figure is available in color online only.

RD, and HMOA in DTI tractography $(\mathrm{p}=0.759,0.759$, $0.400,0.988$, not applicable, respectively) and SD tractography ( $p=0.988,0.759,0.400,0.759,0.759)$ in healthy controls.

\section{Transcranial Magnetic Stimulation Results}

In healthy controls, the median rMT in the left hemisphere (35\%, IQR $\pm 10 \%)$ is statistically similar to that in the right hemisphere $(35 \%, \mathrm{IQR} \pm 9 \% ; \mathrm{p}=0.988)$.

In patients, the median rMT in the hemisphere contralateral to glioma was $40 \%$ (IQR $\pm 15 \%$ ) of the maximum output stimulation. In brain hemispheres with glioma, the median rMT was $46 \%$ (IQR $\pm 17 \%$ ). The rMT in the hemisphere ipsilateral to glioma was significantly greater than that in the contralateral hemisphere (median relative difference $-21.1 \%$, IQR $\pm 14.9 \% ; p=0.038$ ). The rMT contralateral to a glioma was not statistically different from the rMT in healthy subjects $(\mathrm{p}=0.073)$.

Nine of the 10 patients demonstrated a pathological excitability of the motor cortex, with the relative difference in rMT ranging from $12 \%$ to $136 \%$ between the affected and unaffected hemispheres. Only 2 healthy controls had a relative difference in the rMT larger than $10 \%$ between the two hemispheres, but this difference appeared to be modest (11.5\% and 12\%) and was not statistically significant $(\mathrm{p}=0.988)$.

The median mean amplitude in healthy brains was 212 $\mathrm{mV}$ (IQR $\pm 115.65 \mathrm{mV})$. There was no right to left asymmetry in healthy brains $(\mathrm{p}=0.699)$. In the patient group, the median mean amplitude ipsilateral to glioma was 76 $\mathrm{mV}( \pm 88 \mathrm{mV})$ and contralateral to glioma was $123 \mathrm{mV}( \pm$ $46 \mathrm{mV}$ ). There was no significant difference in the mean amplitude of MEPs between brain hemispheres contralateral and ipsilateral to a glioma $(\mathrm{p}=0.297)$.

The mean amplitude of MEPs contralateral to a glioma was statistically smaller than that in healthy controls $(\mathrm{p}=$ 0.001). Similarly, the mean amplitude of MEPs ipsilateral to a glioma was statistically smaller than those in healthy brains $(\mathrm{p}=0.002)$.

The median mean latency of MEPs in a healthy brain was $23 \mathrm{msec}$ (IQR $\pm 1 \mathrm{msec}$ ) on the left and $23 \mathrm{msec}( \pm$ $1.5 \mathrm{msec}$ ) on the right. There was no asymmetry between left and right hemispheres in healthy brains $(\mathrm{p}=0.336)$. The median mean latency of MEPs ipsilateral to a glioma was $24 \mathrm{msec}( \pm 47 \mathrm{msec})$ and contralateral to a glioma was 


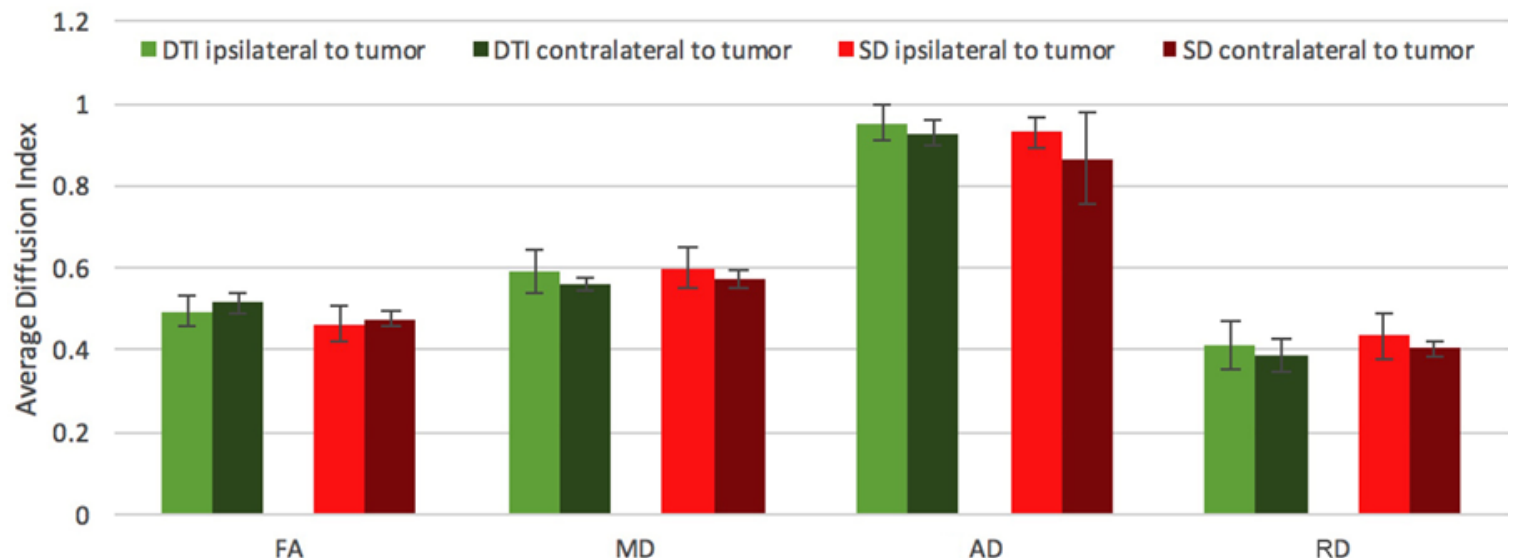

FIG. 2. Bar graph of traditional tractography measurements in the hemisphere ipsilateral to a glioma compared to the those in the contralateral healthy brain hemisphere. Figure is available in color online only.

$24 \mathrm{msec}( \pm 5 \mathrm{msec})$. There was no significant difference in the mean latency of MEPs between brain hemispheres ipsilateral and contralateral to a glioma $(\mathrm{p}=0.518)$. The mean latencies contralateral and ipsilateral to a glioma were not statistically different from those of healthy brains $(\mathrm{p}=0.928)$.

\section{Correlation Between TMS and Tractography}

The higher the rMT ipsilateral to glioma, the lower the HMOA ipsilateral to glioma. The correlation between CST microstructure, as defined by the relative difference between HMOA contralateral and ipsilateral to the glioma, and motor excitability, as defined by the relative difference between rMT contralateral and ipsilateral to the glioma, was also analyzed. The relative difference in HMOA is significantly related to the relative difference in the rMT for the motor cortex contralateral and ipsilateral to glioma. In other words, the degree of microstructure changes seen in the CST (with HMOA) correlates with the degree of functional changes (assessed with rMT; $\mathrm{p}=$ $0.007, \mathrm{r}_{\mathrm{s}}=-0.871 ;$ Fig. 4 ).

In brain hemispheres affected by glioma, $\mathrm{MD}$ and $\mathrm{AD}$ of the CST positively correlated with rMT when SD tractography was used $\left(r_{s}=0.751, p=0.020\right.$ and $r=0.824$, $p$ $=0.006$, respectively; Figs. 5 and 6). MD of the CST was also negatively correlated with latency in healthy brains $\left(\mathrm{r}_{\mathrm{s}}\right.$ $=-0.518, \mathrm{p}=0.028$ ).

\section{Discussion}

It is well known that gliomas can cause changes in the surrounding white matter through a combination of infiltration, edema, and mass effect. ${ }^{29}$ The interaction between gliomas and white matter tracts is of clinical relevance, as it can contribute to the development of neurological deficits. From a neurosurgical perspective, it is mandatory to spare functional white matter tracts, which often constitute the functional boundary of resection identified with intraoperative mapping. ${ }^{30}$ There is extensive literature to suggest that gliomas can change the diffusion profile of white matter tracts, as assessed on preoperative tractography. Several studies have reported that white matter microstructure can be affected by tumors. ${ }^{12,31-33} \mathrm{~A}$ recent meta-analysis on the relation between diffusion metrics and tumor grade in gliomas has suggested the significant effects of tumor grade on average MD and FA. ${ }^{11}$ These changes were more consistently observed in high-grade gliomas, supporting the concept that high-grade tumors are more destructive and infiltrative than low-grade tumors. However, the authors warned against a large heterogeneity in the existing

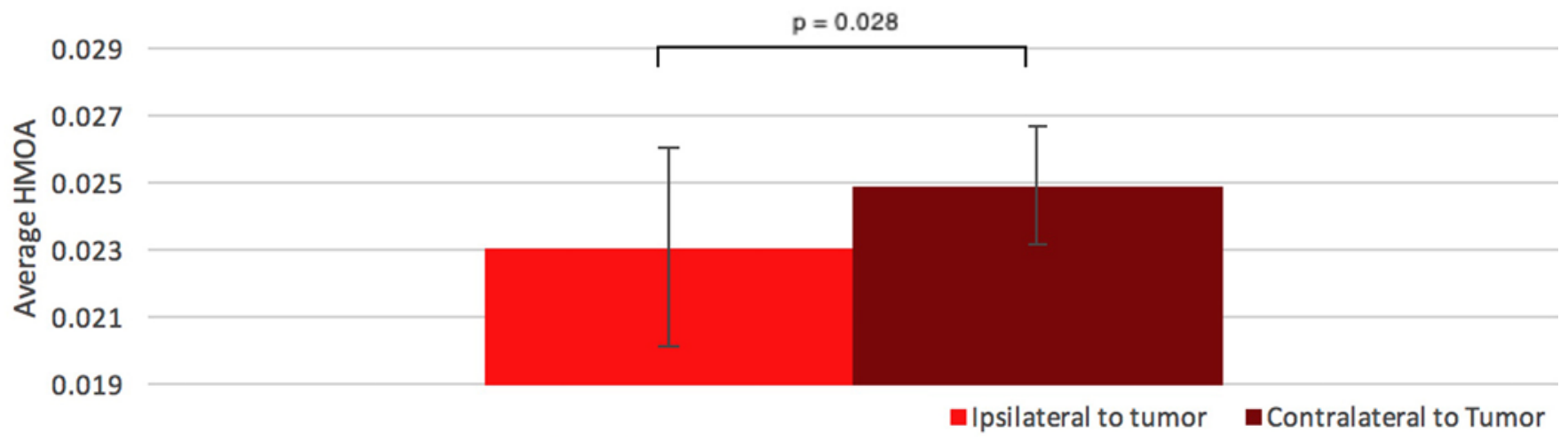

FIG. 3. Bar graph of advanced tractography measurements in the hemisphere ipsilateral to a glioma compared to those in the contralateral healthy brain hemisphere. Figure is available in color online only. 
TABLE 2. Median diffusion indices from SD in healthy brains and in the hemisphere ipsilateral and contralateral to glioma

\begin{tabular}{lcccc}
\hline Index & $\begin{array}{c}\text { Median Value in } \\
\text { Healthy Controls }\end{array}$ & $\begin{array}{c}\text { Median Value } \\
\text { Contralat to Tumor }\end{array}$ & $\begin{array}{c}\text { p Value (healthy controls vs } \\
\text { CST contralat to glioma) }\end{array}$ & $\begin{array}{c}\text { Median Value } \\
\text { Ipsilat to Tumor }\end{array}$ \\
\hline FA & 0.4771565 & 0.476897 & 0.919 & 0.477874 \\
\hline MD & 0.569816 & 0.579547 & 0.152 & 0.577051 \\
\hline AD & 0.896636 & 0.903904 & 0.413 & 0.915615 \\
\hline RD & 0.3991535 & 0.404025 & 0.453 & 0.412706 \\
\hline HMOA & 0.0243325 & 0.0247182 & 0.724 & 0.0231091 \\
\hline
\end{tabular}

literature, especially with regard to tractography methods, tract analysis, and patient populations.

In the present study, we focused on the changes observed in diffusion indices of the CST in a homogeneous population of $I D H$-mutant grade II and III gliomas in close relationship with the central lobule and CST. Diffusion parameters were assessed with both traditional (FA, MD, $\mathrm{AD}$, and $\mathrm{RD}$ ) and advanced (HMOA) indices, and results from the glioma population were compared to those of a healthy population. Our results illustrate that traditional diffusion indices, but not HMOA, are within the healthy range in brain hemispheres ipsilateral to a glioma (i.e., not significantly different compared to healthy subjects or to hemispheres contralateral to a glioma). Traditional diffusion indices (FA, MD, AD, and RD) are based on average voxel characteristics and are therefore susceptible to partial volume effects and crossing fibers. This is particularly relevant for the CST, where there is a significant amount of fiber crossing from callosal and superior longitudinal fasciculus fibers. Additionally, tractography results represent a mathematical reconstruction of the underlying white matter trajectories. Thus, tractography may show partial reconstructions in the presence of a strong partial volume effect due to edema or tumor infiltration. Nevertheless, for this study, we acquired state-of-the-art diffusion imaging data and applied advanced preprocessing and tractogra- phy methods. In particular, the use of high b-values and SD tractography helped to suppress these signal contaminations, resulting in reliable tractography reconstructions consistent across all subjects.

On the other hand, advanced tractography indices, such as HMOA, can be considered true tract-specific indices and can identify characteristics not illustrated by other diffusion indices ${ }^{17}$ In addition, changes in tract-specific characteristics due to factors such as axonal loss, especially in regions of crossing fibers, are better characterized by HMOA. ${ }^{17}$ This may be the reason why only a significant decrease in HMOA is seen in CST trajectories ipsilateral to a glioma. A decrease in HMOA can therefore represent a microstructural change in the CST due to axonal loss. Although more commonly reported in glioblastomas, ${ }^{34}$ changes in traditional diffusion indices have also been found in grade II and III gliomas. ${ }^{12,33}$ However, the $I D H$ status of gliomas was not reported in these papers; therefore, the patient populations are difficult to compare. It is well recognized that $I D H$-mutant and $I D H$-wildtype gliomas differ significantly in terms of biological behavior, aggressiveness, and prognosis. ${ }^{35,36}$ In our homogeneous series of $I D H$-mutant, predominantly grade III gliomas, traditional indices did not show any significant alteration: this may reflect the relatively more indolent behavior of these tumors, requiring advanced (and more sensitive) dif-

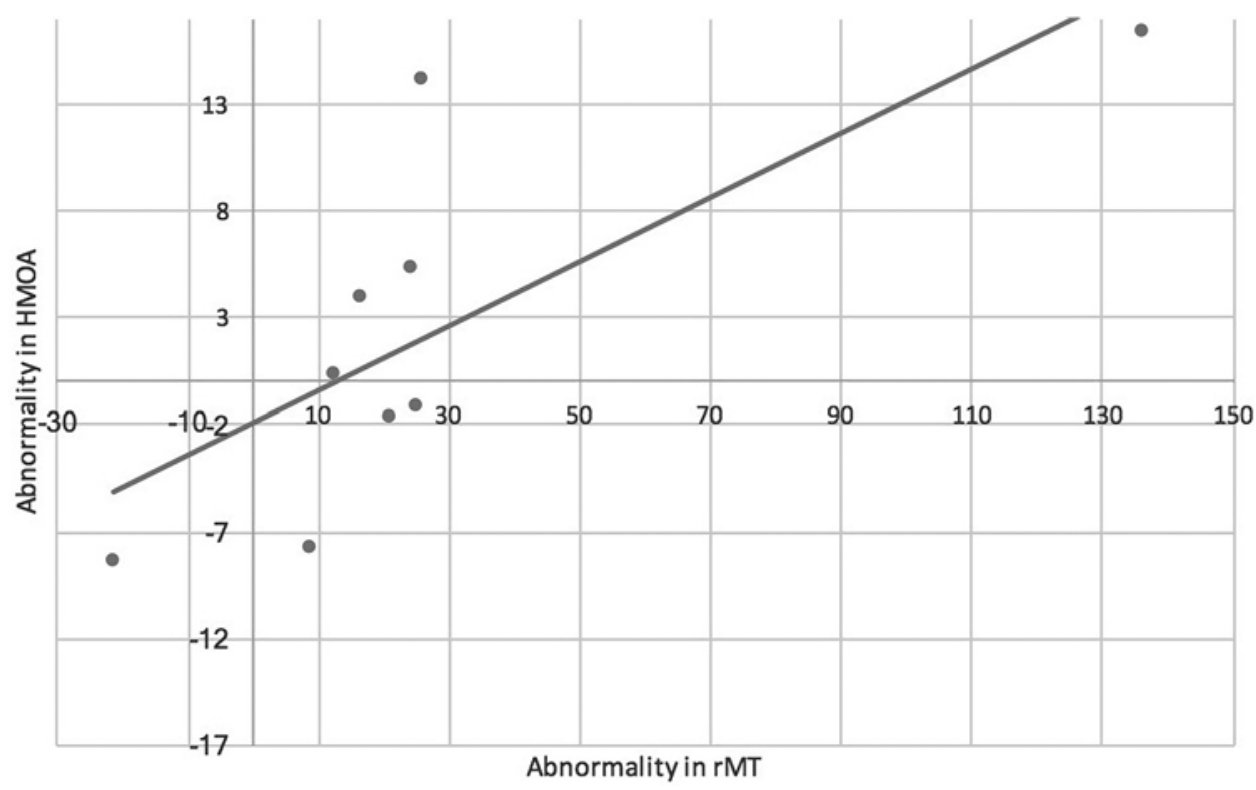

FIG. 4. Correlation between abnormal excitability and abnormal microstructure in glioma patients. 


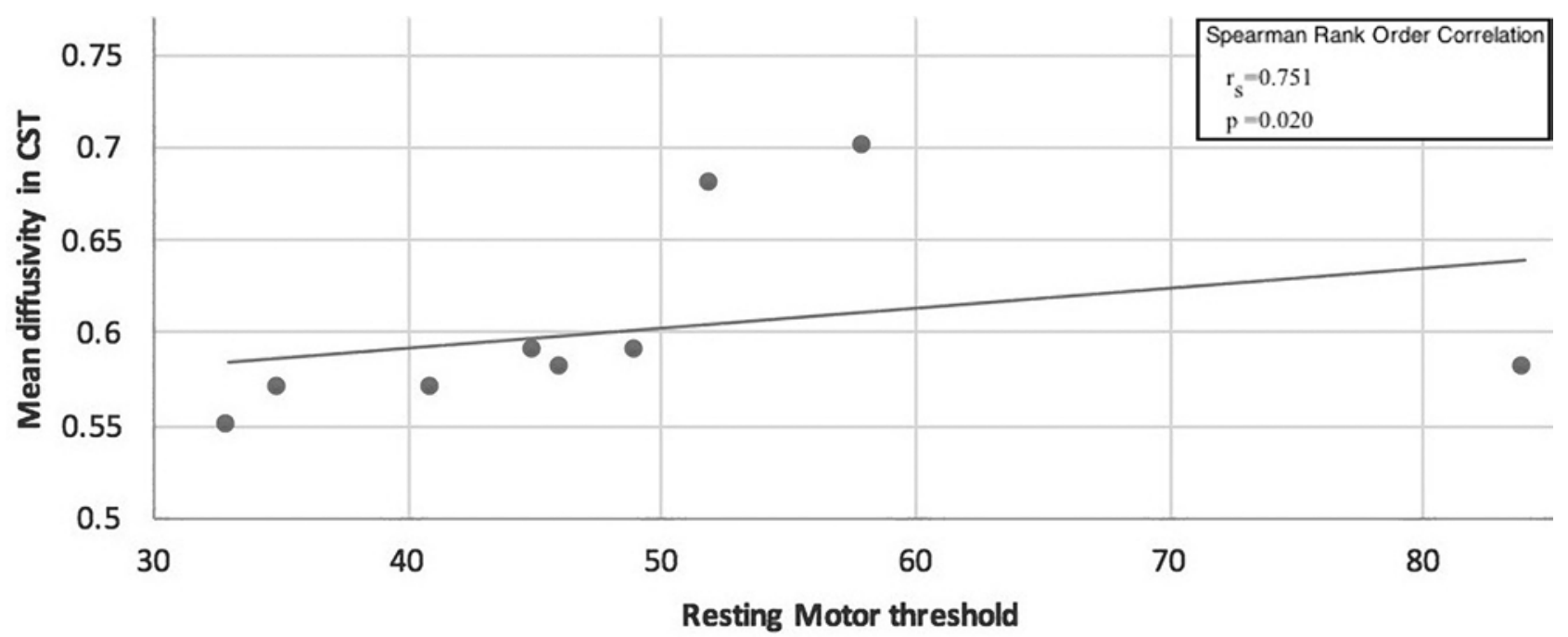

FIG. 5. Correlation between MD in SD (ipsilateral to glioma) and rMT.

fusion parameters to capture microstructural changes at the level of the CST. A larger sample with a more balanced group of grade II and grade III gliomas is needed to address the effect of increased grading on the observed results. It is possible that the observed changes on a microstructural level may be attributable to increased mitotic activity in grade III gliomas.

To further investigate the meaning of the microstructural changes observed in the CST, we attempted to establish whether there is a neurophysiological correlate between changes in the HMOA and measures of cortical excitability of the motor system. To this extent, we used preoperative nTMS as a noninvasive way to assess the motor system in patients and healthy subjects. The advantage of this approach is that, unlike other modalities of noninvasively mapping motor function (such as MEG or fMRI), nTMS has better spatial resolution and allows one to record MEPs for offline analysis. ${ }^{37}$ In addition, unlike direct cortical stimulation, nTMS can explore both hemispheres, permitting interhemispheric comparison in patients (to compare the motor excitability of the pathological versus nonpathological hemisphere) and allowing data to be obtained from healthy volunteers.

Different authors have attempted to establish a relationship between TMS and tractography-derived variables by studying different pathologies and age groups. Hübers et al. failed to demonstrate a relation between rMT and FA in healthy subjects in a voxel-wise analysis..$^{42}$ Jang and colleagues established a correlation between rMT and fiber number in the hemispheres of patients with motor deficits after ischemic vascular injury but failed to show a correlation with the FA values of the CST. ${ }^{38}$ Papadelis et al. studied the evolution of rMT and DTI-derived metrics in children with hemiplegic cerebral palsy and found that the normal correlation of $\mathrm{AD}, \mathrm{MD}$, and $\mathrm{rMT}$ with age was disrupted in these patients. ${ }^{34}$ In the particular case of

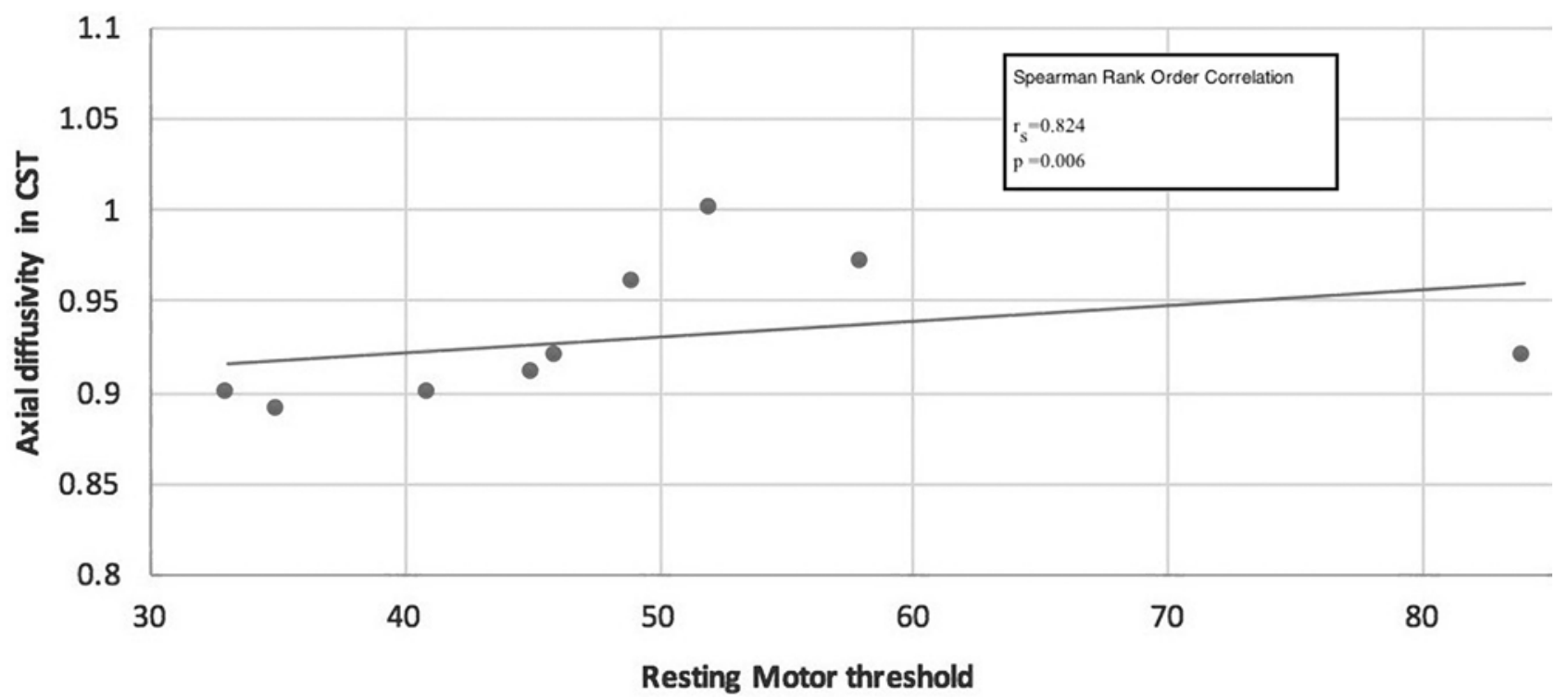

FIG. 6. Correlation between AD in SD (ipsilateral to glioma) and rMT. 
gliomas, Sollmann et al. reported an inverse correlation between rMT and the distance between tumor and CST in patients with permanent or temporary motor deficits after surgery. ${ }^{39}$ However, a clear correlation between nTMS, tractography, and gliomas is missing in the literature.

In our series, the rMT of brain hemispheres with glioma was significantly higher than the rMT of hemispheres contralateral to glioma and that of healthy subjects. In addition, 9 of 10 patients demonstrated a pathological rMT (defined as a difference of more than $10 \%$ between the rMT of the two hemispheres), whereas an rMT difference larger than $10 \%$ was found in only 2 of 10 healthy subjects and with very small and insignificant relative differences. (This latter finding is likely attributable to a margin of error intrinsic to nTMS.) The rMT changes observed in the brain hemisphere with glioma confirm the interaction between glial tumors and motor function, suggesting the presence of a pathological excitability of the motor system in patients.

When SD tractography was used, a significant relationship was found between diffusion indices and rMT. A significant correlation was found between an increase in $\mathrm{MD}$ and $\mathrm{AD}$ and an increase in the rMT in the affected brain hemisphere. In addition, a relation between changes in the CST microstructure and the presence of a pathological motor excitability was found. The higher the rMT ipsilateral to a glioma, the lower the HMOA ipsilateral to a glioma. These results point toward a direct relationship between structural changes in the CST and pathological excitability of the motor system. Different mechanisms can be hypothesized to explain this finding: a loss of axons in the CST, direct tumor invasion, ${ }^{9}$ or distortion and compression on the CST due to mass effect. However, in our series, the presence of peritumoral edema was minimal and diffusion weighting was high, making the latter hypothesis less likely. ${ }^{40}$

It is important to note that all patients were neurologically intact prior to surgery, showing no motor deficits. A correlation between CST changes and pathological rMT may therefore suggest that preclinical changes affecting the motor system can be captured by combining SD tractography and TMS. Risk-stratification models combining imaging and TMS data have been designed with the aim of identifying patients at high risk for developing new motor deficits following surgery. ${ }^{5}$ Our results support the incorporation of tract-specific indices such as HMOA and interhemispheric rMT difference in such risk-stratification models. Information about CST integrity could also be applied when planning the resection of gliomas close to the CST, helping the surgeon in deciding when to stop the resection at the subcortical level in order to prevent damage to the CST itself. ${ }^{41}$ Further research is needed to address these aspects.

\section{Conclusions}

Our results demonstrate that SD tractography reveals the presence of microstructural abnormality within the CST of patients with IDHI-mutant gliomas close to the motor cortex and CST. The microstructural CST changes, captured by the HMOA index, correlate with a pathologi- cal excitability of the motor cortex in neurologically intact patients. The combined use of SD tractography and TMS can provide valuable information on the anatomical-functional preoperative assessment of the motor system.

\section{Acknowledgments}

This paper represents independent research partly funded by the National Institute for Health Research (NIHR) Wellcome Trust King's Clinical Research Facility, the NIHR Maudsley Biomedical Research Centre at South London and Maudsley NHS Foundation Trust, the Wellcome Trust (103759/z/14/z), the Sackler Institute for Translational Neurodevelopment, and King's College London. The views expressed are those of the author(s) and not necessarily those of the NHS, the NIHR, or the Department of Health and Social Care.

We wish to thank all the patients and volunteers who took part in the study.

\section{References}

1. Bello L, Castellano A, Fava E, et al. Preoperative diffusion tensor imaging (DTI): contribution to surgical planning and validation by intraoperative electrostimulation. In: Duffau $\mathrm{H}$, ed. Brain Mapping. Springer; 2011:263-275.

2. Duffau H, ed. Brain Mapping. Springer; 2011.

3. Raabe A, Beck J, Schucht P, Seidel K. Continuous dynamic mapping of the corticospinal tract during surgery of motor eloquent brain tumors: evaluation of a new method. J Neurosurg. 2014;120(5):1015-1024.

4. Gao B, Shen X, Shiroishi MS, et al. A pilot study of preoperative motor dysfunction from gliomas in the region of corticospinal tract: evaluation with diffusion tensor imaging. PLoS One. 2017;12(8):e0182795

5. Rosenstock T, Grittner U, Acker G, et al. Risk stratification in motor area-related glioma surgery based on navigated transcranial magnetic stimulation data. J Neurosurg. 2017;126(4):1227-1237.

6. Essayed WI, Zhang F, Unadkat P, et al. White matter tractography for neurosurgical planning: a topography-based review of the current state of the art. Neuroimage Clin. 2017;15:659672 .

7. Barbosa BJAP, Dimostheni A, Teixeira MJ, et al. Insular gliomas and the role of intraoperative assistive technologies: results from a volumetry-based retrospective cohort. Clin Neurol Neurosurg. 2016;149:104-110.

8. O'Donnell LJ, Rigolo L, Norton I, et al. fMRI-DTI modeling via landmark distance atlases for prediction and detection of fiber tracts. Neuroimage. 2012;60(1):456-470.

9. Catani M, Dell'Acqua F. Mapping white matter pathways with diffusion imaging tractography: focus on neurosurgical applications. In: Duffau H, ed. Brain Mapping. Springer; 2011:61-75.

10. Aung WY, Mar S, Benzinger TL. Diffusion tensor MRI as a biomarker in axonal and myelin damage. Imaging Med. 2013;5(5):427-440.

11. Miloushev VZ, Chow DS, Filippi CG. Meta-analysis of diffusion metrics for the prediction of tumor grade in gliomas. AJNR Am J Neuroradiol. 2015;36(2):302-308.

12. Ormond DR, D'Souza S, Thompson JA. Global and targeted pathway impact of gliomas on white matter integrity based on lobar localization. Cureus. 2017;9(9):e1660.

13. Ille S, Engel L, Kelm A, et al. Language-eloquent white matter pathway tractography and the course of language function in glioma patients. Front Oncol. 2018;8:572.

14. Min ZG, Rana N, Niu C, et al. Does diffusion tensor tractography of the corticospinal tract correctly reflect motor function? Med Princ Pract. 2014;23(2):174-176.

15. Dell'acqua F, Scifo P, Rizzo G, et al. A modified damped 
Richardson-Lucy algorithm to reduce isotropic background effects in spherical deconvolution. Neuroimage. 2010;49(2):1446-1458.

16. Dell'Acqua F, Tournier J. Modelling white matter with spherical deconvolution: how and why? NMR Biomed. 2019;32(4):e3945.

17. Dell'Acqua F, Simmons A, Williams SCR, Catani M. Can spherical deconvolution provide more information than fiber orientations? Hindrance modulated orientational anisotropy, a true-tract specific index to characterize white matter diffusion. Hum Brain Mapp. 2013;34(10):2464-2483.

18. Jung J, Lavrador J-P, Patel S, et al. In reply to the letter to the editor regarding "First United Kingdom experience of navigated transcranial magnetic stimulation in preoperative mapping of brain tumors". World Neurosurg. 2019;125:551-552.

19. Krieg SM, Shiban E, Buchmann N, et al. Utility of presurgical navigated transcranial magnetic brain stimulation for the resection of tumors in eloquent motor areas. J Neurosurg. 2012;116(5):994-1001.

20. Picht T, Mularski S, Kuehn B, et al. Navigated transcranial magnetic stimulation for preoperative functional diagnostics in brain tumor surgery. Neurosurgery. 2009;65(6) (suppl):93-99.

21. Veraart J, Fieremans E, Novikov DS. Diffusion MRI noise mapping using random matrix theory. Magn Reson Med. 2016;76(5):1582-1593.

22. Kellner E, Dhital B, Kiselev VG, Reisert M. Gibbs-ringing artifact removal based on local subvoxel-shifts. Magn Reson Med. 2016;76(5):1574-1581.

23. Andersson JLR, Skare S, Ashburner J. How to correct susceptibility distortions in spin-echo echo-planar images: application to diffusion tensor imaging. Neuroimage. 2003;20(2):870-888.

24. Andersson JLR, Sotiropoulos SN. An integrated approach to correction for off-resonance effects and subject movement in diffusion MR imaging. Neuroimage. 2016;125:1063-1078.

25. Andersson JLR, Graham MS, Drobnjak I, et al. Towards a comprehensive framework for movement and distortion correction of diffusion MR images: within volume movement. Neuroimage. 2017;152:450-466.

26. Kim B, Fisher BE, Schweighofer N, et al. A comparison of seven different DTI-derived estimates of corticospinal tract structural characteristics in chronic stroke survivors. $J \mathrm{Neu}$ rosci Methods. 2018;304:66-75.

27. Kumar A, Juhasz C, Asano E, et al. Diffusion tensor imaging study of the cortical origin and course of the corticospinal tract in healthy children. AJNR Am J Neuroradiol. 2009;30(10):1963-1970.

28. Berger MS, Deliganis AV, Dobbins J, Keles GE. The effect of extent of resection on recurrence in patients with low grade cerebral hemisphere gliomas. Cancer. 1994;74(6):1784-1791.

29. Quail DF, Joyce JA. The microenvironmental landscape of brain tumors. Cancer Cell. 2017;31(3):326-341.

30. Ferracci F-X, Duffau H. Improving surgical outcome for gliomas with intraoperative mapping. Expert Rev Neurother. 2018;18(4):333-341.

31. Liu F, Scantlebury N, Tabori U, et al. White matter compromise predicts poor intellectual outcome in survivors of pediatric low-grade glioma. Neuro Oncol. 2015;17(4):604-613.

32. Mekkaoui C, Metellus P, Kostis WJ, et al. Diffusion tensor imaging in patients with glioblastoma multiforme using the supertoroidal model. PLoS One. 2016;11(1):e0146693.

33. Miller P, Coope D, Thompson G, et al. Quantitative evaluation of white matter tract DTI parameter changes in gliomas using nonlinear registration. Neuroimage. 2012;60(4):23092315.

34. Papadelis C, Kaye H, Shore B, et al. Maturation of corticospinal tracts in children with hemiplegic cerebral palsy assessed by diffusion tensor imaging and transcranial magnetic stimulation. Front Hum Neurosci. 2019;13:254.

35. Eckel-Passow JE, Lachance DH, Molinaro AM, et al. Glioma groups based on 1p/19q, IDH, and TERT promoter mutations in tumors. N Engl J Med. 2015;372(26):2499-2508.

36. Yan H, Parsons DW, Jin G, et al. IDH1 and IDH 2 mutations in gliomas. N Engl J Med. 2009;360(8):765-773.

37. Frey D, Strack V, Wiener E, et al. A new approach for corticospinal tract reconstruction based on navigated transcranial stimulation and standardized fractional anisotropy values. Neuroimage. 2012;62(3):1600-1609.

38. Jang SH, Kim DH, Kim SH, Seo JP. The relation between the motor evoked potential and diffusion tensor tractography for the corticospinal tract in chronic hemiparetic patients with cerebral infarct. Somatosens Mot Res. 2017;34(2):134-138.

39. Sollmann N, Wildschuetz N, Kelm A, et al. Associations between clinical outcome and navigated transcranial magnetic stimulation characteristics in patients with motor-eloquent brain lesions: a combined navigated transcranial magnetic stimulation-diffusion tensor imaging fiber tracking approach. J Neurosurg. 2018;128(3):800-810.

40. Acampora A, Manzo G, Fenza G, et al. High b-value diffusion MRI to differentiate recurrent tumors from posttreatment changes in head and neck squamous cell carcinoma: a single center prospective study. Biomed Res Int. 2016;2016:2865169.

41. Lavrador JP, Ghimire P, Brognam C, et al. Pre and intraoperative mapping for lesions in primary motor cortex: decision making process in surgical resection. Poster presented at: EANS2019; September 24-28, 2019; Dublin, Ireland.

42. Hübers A, Klein JC, Kang J-S, et al. The relationship between TMS measures of functional properties and DTI measures of microstructure of the corticospinal tract. Brain Stimul. 2012;5(3):297-304.

\section{Disclosures}

The authors report no conflict of interest concerning the materials or methods used in this study or the findings specified in this paper.

\section{Author Contributions}

Conception and design: Mirchandani, Dell'Acqua, Vergani. Acquisition of data: Mirchandani, Beyh, Howells, Dell'Acqua. Analysis and interpretation of data: Mirchandani, Beyh, Dell'Acqua, Vergani. Drafting the article: Mirchandani, Lavrador, Vergani. Critically revising the article: Mirchandani, Dell'Acqua, Vergani. Reviewed submitted version of manuscript: Mirchandani, Lavrador, Dell'Acqua, Vergani. Approved the final version of the manuscript on behalf of all authors: Mirchandani. Statistical analysis: Mirchandani. Administrative/technical/material support: Mirchandani, Dell'Acqua. Study supervision: Dell'Acqua, Vergani.

\section{Supplemental Information \\ Previous Presentations}

Portions of this work were presented in abstract form as proceedings at the Society of British Neurological Surgeons Spring Meeting held in Manchester, England, on March 19, 2019, and at the 19 th European Congress of Neurosurgery held in Dublin, Ireland, on September 24, 2019.

\section{Correspondence}

Ayesha Sunil Mirchandani: King's College London, United Kingdom.mirchandani.ayesha@gmail.com. 